\title{
Early time evolution of negative ion clouds and electron density depletions produced during electron attachment chemical release experiments
}

\author{
W. A. Scales \\ Bradley Department of Electrical Engineering, Virginia Tech, Blacksburg, Virginia
}

\section{P. A. Bernhardt and G. Ganguli}

Space Plasma Branch, Plasma Physics Division, Naval Research Laboratory, Washington D.C.

\begin{abstract}
Two-dimensional electrostatic particle-in-cell simulations are used to study the early time evolution of electron depletions and negative ion clouds produced during electron attachment chemical releases in the ionosphere. The simulation model considers the evolution in the plane perpendicular to the magnetic field and a three-species plasma that contains electrons, positive ions, and also heavy negative ions that result as a by-product of the electron attachment reaction. The early time evolution (less than the negative ion cyclotron period) of the system shows that a negative charge surplus initially develops outside of the depletion boundary as the heavy negative ions move across the boundary. The electrons are initially restricted from moving into the depletion due to the magnetic field. An inhomogenous electric field develops across the boundary layer due to this charge separation. A highly sheared electron flow velocity develops in the depletion boundary due to $\mathbf{E} \times \mathbf{B}$ and $\nabla N \times \mathbf{B}$ drifts that result from electron density gradients and this inhomogenous electric field. Structure eventually develops in the depletion boundary layer due to low-frequency electrostatic waves that have growth times shorter than the negative ion cyclotron period. It is proposed that these waves are most likely produced by the electron-ion hybrid instability that results from sufficiently large shears in the electron flow velocity.
\end{abstract}

\section{Introduction}

A problem that is similar in many respects to the release of neutral chemicals that photoionize in the Earth's ionosphere, such as barium, cesium, sodium, europium, and lithium, is the release of electron attachment chemicals. Initially, when released into the $F$ region, these chemicals attach electrons and produce electron density depletions. Heavy negative ion clouds are created as a by-product of the electron attachment reaction. At later times, the background positive ion density (composed primarily of $\mathrm{O}^{+}$at the altitudes of interest) will be reduced by mutual neutralization with the heavy negative ions. The mutual neutralization reaction provides a useful ground-based optical diagnostic tool since the reaction products are left in electronically excited states [Bernhardt et al., 1986]. Two important advantages of the electron attachment chemical release over the photoionization chemical release is that (1) no sunlight is required for the chemical reactions to take place and (2) a depletion rather than an enhancement

Copyright 1994 by the American Geophysical Union.

Paper number 93JA02752.

0148-0227/94/93JA-02752\$05.00 in the ionospheric plasma density is created. Calculations by Bernhardt et al. [1991] have predicted that electron attachment chemicals produce faster, larger, and longer lasting perturbations to the ionosphere than typical photoionization chemicals. This is because of the larger reaction rate constants of the electron attachment materials. The largest electron density gradients, ion fluxes, and polarization electric fields should be produced by electron attachment chemical releases. Important uses for the electron attachment release may be the perturbation of auroral current systems [Bernhardt et al., 1991], focusing of high-power radio waves [Bernhardt and Duncan, 1987a], and the study of the physics of negative ion plasmas in space [Ganguli et al., 1993]. Typical chemicals used during recent experiments are sulfur hexafluoride $\left(\mathrm{SF}_{6}\right)$, trifloromethly bromide $\left(\mathrm{CF}_{3} \mathrm{Br}\right)$, and nickel carbonyl $\left(\mathrm{Ni}(\mathrm{CO})_{4}\right)$. The dissociative electron attachment reactions for these materials are as follows:

$$
\begin{gathered}
\mathrm{SF}_{6}+e^{-} \rightarrow \mathrm{SF}_{5}^{-}+\mathrm{F} \\
\mathrm{CF}_{3} \mathrm{Br}+e^{-} \rightarrow \mathrm{Br}^{-}+\mathrm{CF}_{3} \\
\mathrm{Ni}(\mathrm{CO})_{4}+e^{-} \rightarrow \mathrm{Ni}(\mathrm{CO})_{3}^{-}+\mathrm{CO}
\end{gathered}
$$


In each case, heavy negative ions are created whose mass is much larger than the background $\mathrm{O}^{+}$mass. The studies by Bernhardt [1987b] and Bernhardt et al. [1991] have shown that $\mathrm{Ni}(\mathrm{CO})_{4}, \mathrm{CF}_{3} \mathrm{Br}$, and $\mathrm{SF}_{6}$ are in many ways superior to chemicals that have been historically used to create electron depletions by ion-molecule reactions such as $\mathrm{H}_{2}$ and $\mathrm{H}_{2} \mathrm{O}$. This is because of the large reaction rate constants of the electron attachment materials.

In the past decade, there have been several theoretical and numerical studies of processes associated with electron attachment chemical releases. Theoretical studies have considered electron attachment and neutralization chemistry, airglow production, ambipolar electric fields along the geomagnetic field, and macroscopic plasma instabilities [Mendillo and Forbes, 1982; Bernhardt, 1984, $1986,1987 \mathrm{~b}]$. The first nonlinear numerical simulation of the electron attachment release was the twodimensional work of Bernhardt [1988]. This work used an electrostatic fluid model to study the macroscopic processes associated with the motion of the electron depletion and negative ion cloud across the geomagnetic field when under the influence of a neutral windgenerated electric field. This work predicted the structuring of the plasma depletion by the $\mathbf{E} \times \mathbf{B}$ interchange instability. Recent simulation works by Bernhardt et al. [1991] and Scales and Bernhardt [1991] have considered the electrodynamics of the releases along the geomagnetic field lines and structuring of the depletion due to high-speed release of the chemicals at satellite velocities across the geomagnetic field. These works, like the work of Bernhardt [1988], used macroscopic fluid simulation models and considered the evolution on large space and timescales.

A recently conducted series of sounding rocket experiments called the Nickel Carbonyl Release Experiments (NICARE) have been able to study the very early time microscopic processes associated with the release of electron attachment chemicals for the first time [Bernhardt et al., 1991, 1993; Ganguli et al., 1993]. These experiments released $\mathrm{CF}_{3} \mathrm{Br}$ and $\mathrm{Ni}(\mathrm{CO})_{4}$ at altitudes of $300-400 \mathrm{~km}$ at midlatitudes and were successful at creating electron density depletions. A Langmuir probe and VLF plasma wave receiver were used to obtain the first in situ measurements of electron density and electric field fluctuations during an electron attachment chemical release. Results of the NICARE 1 experiment [Bernhardt et al., 1991; Ganguli et al., 1993] showed that during the release of $30 \mathrm{~kg}$ of $\mathrm{CF}_{3} \mathrm{Br}$, the electron density was reduced from $10^{5} \mathrm{~cm}^{-3}$ to $15 \mathrm{~cm}^{-3}$ in less than $0.1 \mathrm{~s}$. Large electron density gradients were observed that initially had scale lengths less than 100 $m$ which compares to an $\mathrm{O}^{+}$Larmor radius of the order of $10 \mathrm{~m}$. Therefore the modified ionosphere could be considered as an ambient ionospheric plasma $\left(e^{-}-\mathrm{O}^{+}\right)$ separated from a negative ion plasma $\left(\mathrm{Br}^{-}-\mathrm{O}^{+}\right)$by a relatively thin boundary layer. The NICARE 1 plasma wave receiver detected VLF waves that were coincident with the formation of the electron depletion and negative ion cloud. At early times $(<5 \mathrm{~s})$, when the de- tector was near the depletion boundary layer, waves near $200 \mathrm{~Hz}$ were observed. This compares to a lower hybrid frequency of several kilohertz. At later times, when the detector was further inside the negative ion plasma, waves at frequencies between 2 and $10 \mathrm{~Hz}$ were observed. These waves were possibly near the $\mathrm{Br}^{-}$and $\mathrm{Ni}(\mathrm{CO})_{3}^{-}$cyclotron frequencies which were roughly 9 and $5 \mathrm{~Hz}$, respectively.

These new observations during NICARE have motivated us to develop a numerical simulation model to study the very early time microscopic processes observed during the releases. In this paper we report on an initial attempt at modeling the early time evolution of the electron depletion and negative ion cloud as well as the development of low-frequency electrostatic waves. Section 2 describes the simulation model in detail. Section 3 describes results from a representative simulation run. The initial evolution of the electron depletion and negative ion cloud and the development of electrostatic plasma waves in the depletion boundary layer are emphasized. A summary of the results and a discussion are presented in section 4.

\section{Simulation Model}

To model the early time behavior of the electron density depletion, negative ion cloud, and electrostatic waves observed during the NICARE experiments, we have developed a two-space and three-velocity dimension periodic electrostatic particle-in-cell simulation code by using standard techniques [Hockney and Eastwood, 1988; Tajima, 1989; Birdsall and Langdon, 1991]. Our simulation model considers the evolution in the plane perpendicular to the geomagnetic field and a low beta plasma. The simulation model allows us to study processes on timescales of the order of or less than the negative ion cyclotron period which corresponds to a few one-tenths of seconds during the experiments. Our twodimensional approximation is valid on this timescale since the work of Bernhardt et al. [1991] shows that the electron depletion lifetime along the magnetic field is at least a few tens of seconds. Large ambipolar electric fields sustain the steep field-aligned electron density gradient and prevent electrons from filling the depletion in during this time period. Of course, the electron density gradient in the plane perpendicular to the magnetic field is sustained by the cyclotron motion of the electrons. Therefore the electron hole can be considered in a plane perpendicular to the magnetic field. The spatial scales to be considered are hundreds of meters in accordance with the steep early time density gradients observed during the NICARE experiment.

We consider a three-species magnetized plasma that consists of heavy negative ions, $\mathrm{X}^{-}$, positive ions, $\mathrm{O}^{+}$, and electrons, $e^{-}$. The mass ratios are $m_{X} / m_{e}=400$ and $m_{0} / m_{e}=50$. The choosen mass ratio between the positive ions and electrons is sufficient to separate the ion and electron timescales and allows the simulation to be run with a reasonable amount of CPU time while following the full electron dynamics. The mass ratio 
of the negative ion species discussed earlier $\left(\mathrm{SF}_{5}^{-}, \mathrm{Br}^{-}\right.$, and $\mathrm{Ni}(\mathrm{CO})_{3}^{-}$) and $\mathrm{O}^{+}$is approximately 8,5 , and 9 , respectively. The mass ratio of the negative and positive ions in the simulation is $m_{\mathbf{X}} / m_{0}=8$ which is representative of these values. The chemical reactions that occur during the release directly determine the density gradient scale lengths in the boundary layer and indirectly determine the dc electric field that results from charge imbalance near the boundary. In our present model, we neglect the complexities of the chemical reactions, in particular, the electron attachment, and assume that electrons have been attached prior to the initialization of the simulation. Note that this does not necessarily imply that all of the neutrals have attached electrons prior to initialization. On the early timescales we consider, only a small fraction of the total number of neutrals have attached electrons. However, because of the high neutral density on this timescale, this small fraction of neutrals may be enough to totally deplete the electron density in a sufficiently small localized region. We have developed a more sophisticated model that incorporates electron attachment and the results of how chemistry affects plasma processes will be reported on in the near future.

Figure 1 shows a cross section of the densities of the three particle species in our simulation at initialization. The simulation box is $128 \times 128$ grid cells, where the grid cell size is equal to the ambient electron Debye length $\lambda_{\mathrm{De}}$. We use 20 simulation particles per grid cell for each species. The electrons are initialized with a density depletion in which there are no electrons within a circular disk region at the center of the simulation box. The negative ions populate this circular disk region, while the positive ion density is constant throughout the simulation box. This configuration guarantees charge neutrality and zero electrostatic energy at initialization. The importance of the mutual neutralization of the negative and positive ions may be neglected as a result of the short timescales we are considering. The electron depletion has a radius of 32 grid cells (or

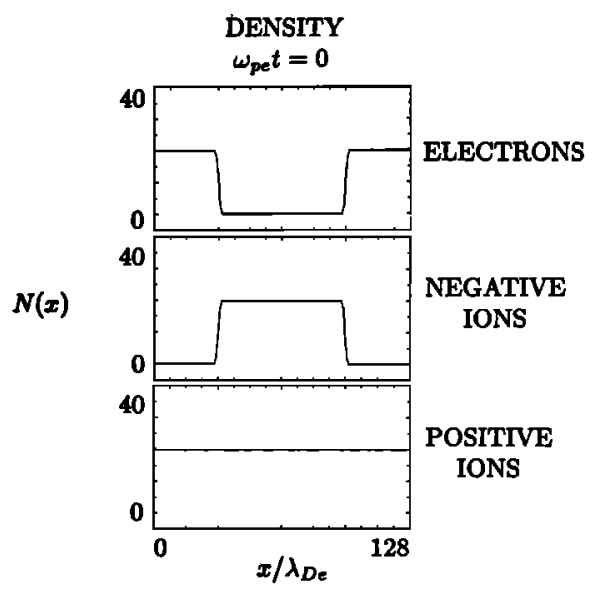

Figure 1. A cross - section of the initial particle densities in the simulation model. Units of the density are particles per grid cell. Note that the electron density is zero inside the depletion. $\left.\lambda_{\text {De }}\right)$. All particles are loaded with standard Maxwellian velocity distributions and have zero-directed velocity. After initialization, the particles are allowed to freely move across the boundary because of their initial thermal energy. For the simulation results that we discuss, the ratio of the ambient electron plasma frequency to the electron cyclotron frequency, $\omega_{\mathrm{pe}} / \Omega_{\mathrm{ce}}$, is 0.5 . The temperature of the three species is taken to be equal, that is, $T_{e}=T_{X}=T_{O}$. With the chosen values of particle temperatures and cyclotron frequencies, the Larmor radii of the electrons, negative and positive ions moving at the thermal velocity are $\rho_{e}=0.5 \lambda_{\mathrm{De}}, \rho_{\mathrm{X}}=10 \lambda_{\mathrm{De}}$, and $\rho_{\mathrm{O}}=3.5 \lambda_{\mathrm{De}}$, respectively. In this case, the radius of the electron depletion is $3.2 \rho \mathrm{x}$. As we stated earlier, the results of one simulation run with the parameters previously stated will be presented and discussed in detail. The ratios $m_{e} / m_{0}, m_{\mathbf{X}} / m_{0}, \omega_{\mathrm{pe}} / \Omega_{\mathrm{ce}}$, and $T_{e} / T_{\mathbf{X}}$ and the depletion radius have been varied in a number of other simulation runs producing qualitatively similar results. The important effects of varying several of these parameters will be briefly discussed afterward.

\section{Results}

The simulation model just described was used to study the nonlinear early time evolution of the electron depletion and negative ion cloud produced by an electron attachment chemical release. The simulation was run to the time $\omega_{\mathrm{pe}} t=1000$ which is roughly 0.8 negative ion cyclotron periods or 20 ambient lower hybrid periods where the ambient lower hybrid frequency $\omega_{\mathrm{LH}}$, is

$$
\omega_{\mathrm{LH}}^{2}=\frac{\omega_{\mathrm{O}}^{2}}{1+\left(\omega_{\mathrm{pe}} / \Omega_{\mathrm{ce}}\right)^{2}}
$$

with wo the ambient $\mathrm{O}^{+}$plasma frequency. Note that the lower hybrid frequency in the boundary layer, where both ion species are present, will be given by $\omega_{\mathrm{LH}}^{2}=$ $\left(\omega_{\mathrm{o}}^{2}+\omega_{\mathrm{X}}^{2}\right) /\left(1+\left(\omega_{\mathrm{pe}} / \Omega_{\mathrm{ce}}\right)^{2}\right)$, where $\omega_{\mathrm{X}}$ is the negative ion plasma frequency. Since $m_{\mathrm{X}} \gg m_{\mathrm{O}}$ and therefore $\omega_{0}^{2} \gg \omega_{X}^{2}$, the lower hybrid frequency in the boundary layer is approximately equal to that in the ambient plasma. First, we describe the initial dynamics of the system and afterward the development of electrostatic waves in the depletion boundary layer.

\subsection{Initial Dynamics}

The early evolution in the simulation shows that the heavy negative ions move across the depletion boundary due to their initial thermal energy since they are essentially unmagnetized on the simulation timescales. The electrons are strongly magnetized, and the magnetic field restricts their motion across the boundary. This produces an excess of negative charge outside of the boundary and an outward electric field, $E_{r}$, develops in the radial, $\hat{\mathbf{r}}$, direction. This field is localized at the depletion boundary. Figure 2 shows the angle averaged densities of the three particle species at two times during the simulation run. At the early time, $\omega_{\mathrm{pe}} t=300$, the electrons have been unable to move 


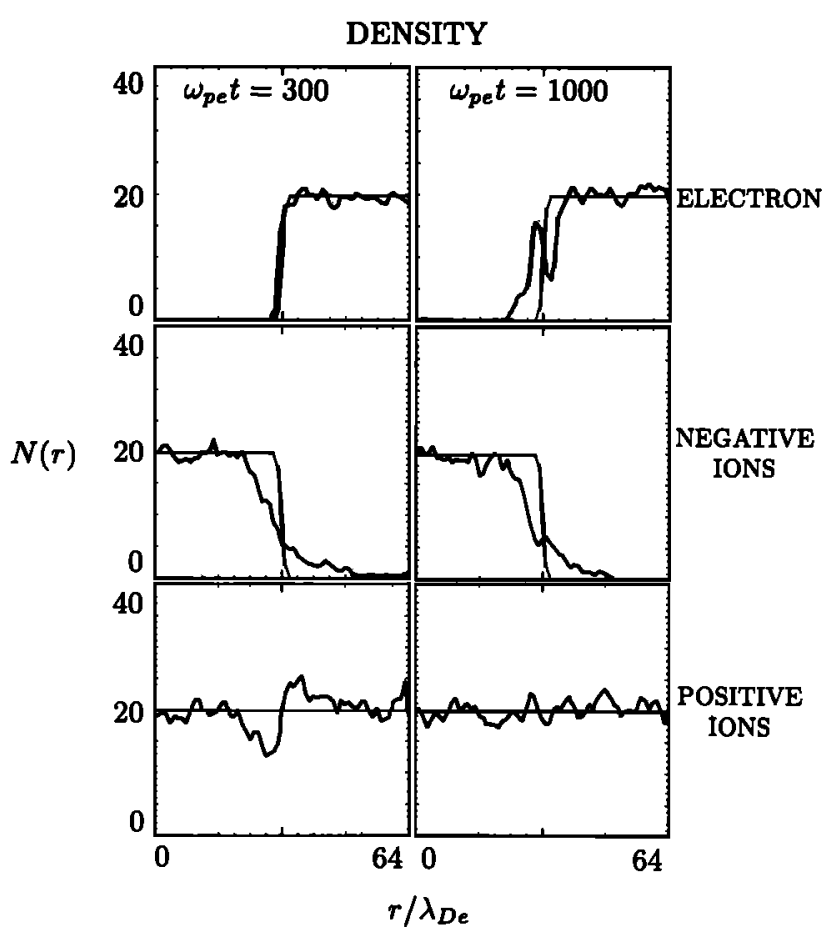

Figure 2. Particle density profiles in the simulation run at $\omega_{\mathrm{pe}} t=300$ and $\omega_{\mathrm{pe}} t=1000$. Light line indicates the densities at $\omega_{\mathrm{pe}} t=0$. Note the negative ions diffuse across the boundary at early times and the electrons diffuse into the depletion at late times.

across the boundary into the depletion due to the magnetic field. The negative ions have moved across the boundary to create a surplus of negative charge outside the depletion. Also, note that the positive ion density exhibits a depression near the boundary. This is due to acceleration of the positive ions across the depletion by the radial ambipolar electric field before they begin their cyclotron motion. At later times, $\omega_{\mathrm{pe}} t=1000$, the electrons have diffused into the depletion due to anomalous processes caused by the development of electrostatic waves in the depletion boundary layer. This will be discussed in more detail shortly.

An azimuthally directed, $\hat{\theta}, \mathbf{E} \times \mathbf{B}$ flow velocity may develop in each particle species due to the radial electric field. Also, because of the radial density gradients at the boundary, azimuthal diamagnetic drifts $(\nabla N \times \mathbf{B})$ may result. The total azimuthal flow velocity of a particular particle species, $V_{\theta s}$, will be made up of these two components and is given by

$$
V_{\theta s}(r)=-\frac{E_{r}(r)}{B}+\frac{T_{s}}{q_{s} B} \frac{1}{N_{s}(r)} \frac{\partial N_{s}(r)}{\partial r}
$$

Here the particle species charge, density, and temperature are denoted by $q_{s}, N_{s}$, and $T$, respectively. The first term in (2) is the $\mathbf{E} \times \mathbf{B}$ drift, $V_{E}(r)$, and it may be related to the particle densities through Poisson's equation as

$$
\frac{\partial V_{E}(r)}{\partial r}=\frac{1}{B} \frac{\partial E_{r}(r)}{\partial r}=e\left(N_{O}(r)-\left(N_{X}(r)+N_{e}(r)\right)\right) / \epsilon_{0}
$$

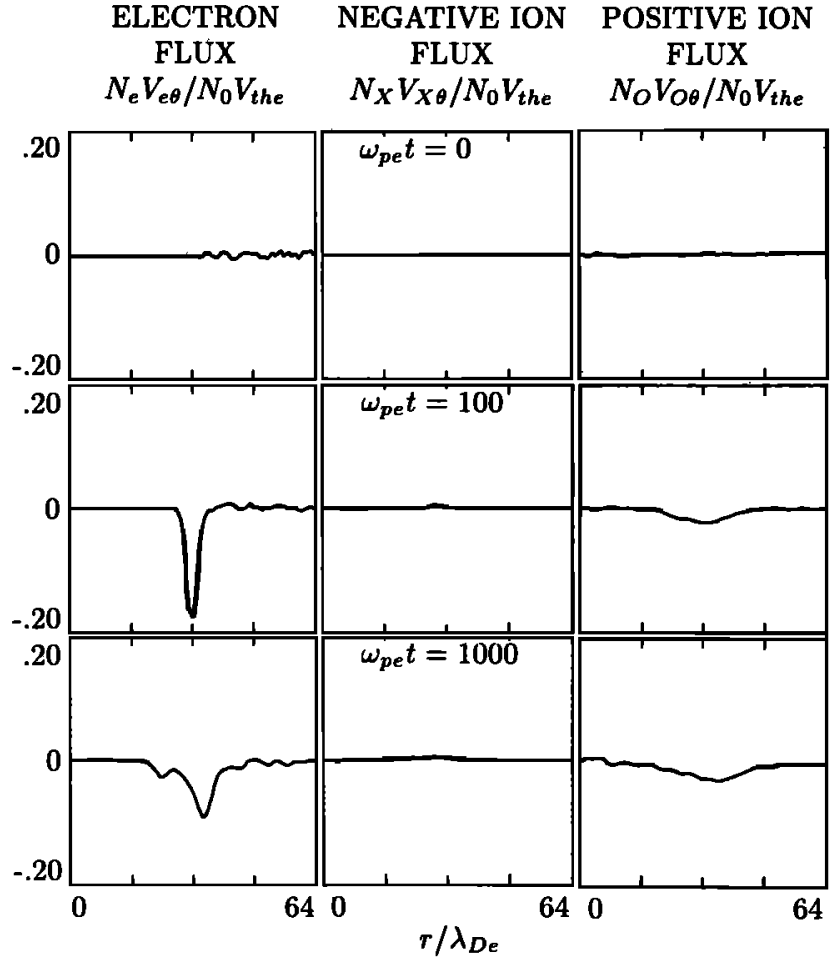

Figure 3. Azimuthal fluxes $\left(N V_{\theta}\right)$ of the three species at $\omega_{\text {pe }} t=0,100$, and 1000 . The fluxes are normalized to $N_{0} V_{\text {the }}$ where $N_{0}$ is the ambient particle density and $V_{\text {the }}$ is the electron thermal velocity. Note that the electron flux is much larger than that of the ions and is sheared at $\omega_{\mathrm{pe}} t=100$. At the end of the run $\left(\omega_{\mathrm{pe}} t=\right.$ 1000) the electron flux has decreased significantly.

where $e$ is the unit charge and $\epsilon_{0}$ is the permittivity constant. Since the particle densities depend on the radial distance, $r$, both the $\mathbf{E} \times \mathbf{B}$ and $\nabla N \times \mathbf{B}$ flow velocities will be sheared. Also note that the $\mathbf{E} \times \mathbf{B}$ and diamagnetic drifts will be in the same direction for the electrons and in opposite directions for the negative ions.

Figure 3 shows the azimuthal flux $\left(N V_{\theta}\right)$ of the three particle species at three times during the simulation run. (Note that these profiles have been averaged in the azimuthal direction.) At $\omega_{\mathrm{pe}} t=100$, the electron flux can be seen to be much larger than the ion fluxes. The electron flux is also highly sheared and localized near the depletion boundary $\left(r / \lambda_{D e}=32\right)$. By the end of the run, the electron flux profile is seen to significantly broaden. There is also a shift of the maximum flux to a radius larger than the initial depletion radius. Throughout the run, the fluxes of the ion species remain small in comparison to the electron flux since the ions are weakly magnetized. The negative ion flux is observed to be in the opposite direction to the postive ion and electron flux.

The temporal evolution of the electrostatic field energy and average azimuthal flow velocity of each species (averaged over all particles of that species) is shown in Figure 4. The total electric field is composed of the radial component, $E_{r}$, that results from the radial charge separation and an azimuthal component, $E_{\theta}$, that ulti- 
mately results from plasma instabilities driven by the density and flow velocity gradients. The energy in these two components of the field (normalized to the initial negative ion kinetic energy) is shown separately. The radial electrostatic energy grows above the noise level and reaches a maximum around $\omega_{\mathrm{pe}} t=300$ and then slowly decreases. The azimuthal field energy, which is smaller than the radial energy, grows relatively rapidly during the time the radial energy grows. A slow increase in the azimuthal energy is observed after that time. The average azimuthal flow velocities maximize around the same time that the radial electrostatic energy does and begin to decrease by the end of the run. The average azimuthal flow velocity for the electrons is larger than that for the ion species. Again, we see that the average negative ion flow velocity is in the opposite direction to the electrons and positive ions. Note that the average flow velocities are small in comparison to the electron thermal velocity $v_{\text {the }}$ and also smaller than the negative ion thermal velocity in this case. The maximum value for the electron flow shown in Figure 4 is roughly $0.6 v_{\text {th }}$.

The energy for the electrostatic fields shown in Figure 4 ultimately comes from the motion of the negative ions as they move across the depletion boundary due to their initial kinetic energy. Figure 5 shows the kinetic energy of the ion species (normalized to the initial negative ion kinetic energy) during the simulation run. The negative ions initially decelerate as they move across the boundary and their total kinetic energy minimizes at the same time the total electrostatic energy maximizes. Because of the development of the radial electric field, the positive ions are accelerated across the boundary, and their total kinetic energy initially increases as can be seen in Figure 4. At later times, the positive ion kinetic energy decreases and the negative ion kinetic energy increases as the total electrostatic energy decreases. The total

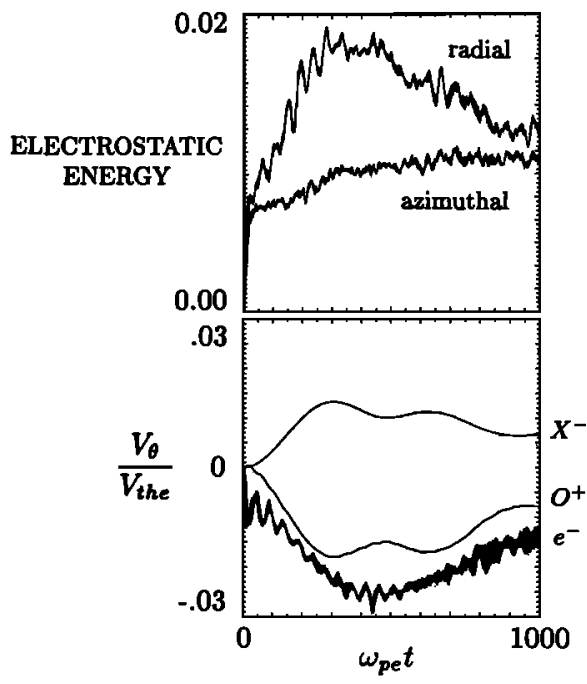

Figure 4. Radial and azimuthal electrostatic field energy (normalized to the initial total negative ion kinetic energy $n_{\mathrm{X}} T_{\mathrm{X}}$ ) and the average azimuthal flow velocities (averaged over all particles of the species) of the three particles species during the simulation. kinetic energy loss by the negative ions goes into the electrostatic energy and the acceleration of the positive ions across the boundary. The total electron kinetic energy change (not shown) is neglibly small during the run.

\subsection{Wave Development}

As we discussed earlier, electrostatic waves associated with the azimuthal electric field develop in the depletion boundary layer and propagate azimuthally around the depletion boundary. These waves which begin to develop early in the run, can initially be seen around $\omega_{\text {pe }} t$ $=100$ and continue to grow until roughly $\omega_{\mathrm{pe}} t=400$. Figure 6 shows the radial and azimuthal electric fields at $\omega_{\mathrm{pe}} t=300$ which is near the time the total electrostatic energy maximizes in Figure 4 . We see that the radial electric field is highly localized near the boundary of the depletion $\left(r / \lambda_{\mathrm{De}}=32\right)$. The azimuthal electric field at the boundary in Figure 6 exhibits coherent structure with the wavelength roughly $9 \lambda_{D e}$ which is less than the negative ion Larmor radius.

To consider the effect of development of the waves on the density of the plasma species, we show in Figures 7 and 8 the electrostatic potential and the density of each plasma species in two-dimensional space. These quantities are shown at three times, $\omega_{\mathrm{pe}} t=100,400$, and 1000. At $\omega_{\mathrm{pe}} t=100$, Figure 7 shows the potential is localized near the depletion boundary. At $\omega_{\text {pe }} t=400$, the potential exhibits structure as a result of the development of the waves and has increased in magnitude by roughly a factor of 2 . At the end of the run, the potential has broadened in space, and its magnitude has reduced to a value near to that at $\omega_{\text {pe }} t=100$. Figure 8 shows the initial development of the waves in the electron density at $\omega_{\mathrm{pe}} t=100$. Later, at $\omega_{\mathrm{pe}} t=400$ and 1000 , the structure is more pronounced, and close inspection shows the development of vortices. Because of anomalous diffusion of the electrons into the depletion

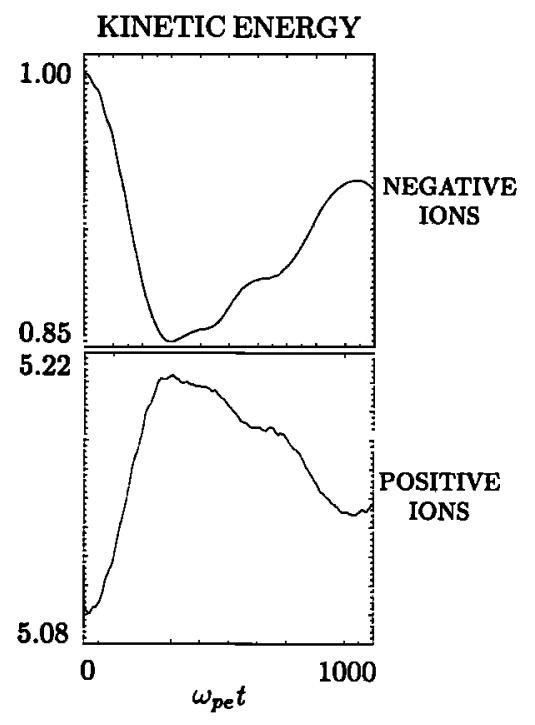

Figure 5. Kinetic energy of the positive and negative ions (normalized to the initial total negative ion kinetic energy, $\left.n_{X} T_{X}\right)$ during the run. 

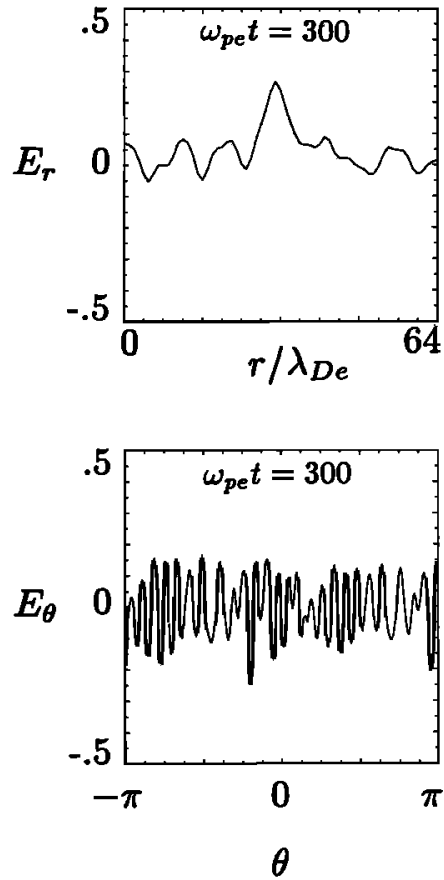

Figure 6. Radial and azimuthal electric fields at $\omega_{\text {pe }} t$ $=300$ (normalized to $m_{e} v_{\text {the }} \omega_{\text {pe }} / q$ ). Note the radial field is localized near the depletion boundary $\left(r / \lambda_{\mathrm{De}}=\right.$ 32).

caused by the development of waves, the initial sharp electron density gradient relaxes significantly by the end of the run. This can be seen clearly in Figure 2 as well. The diffusion of the negative charge into the depletion ultimately reduces the radial component of the electric field as shown in Figure 4. The structure in the negative and positive ion densities is less pronounced than the structure in the electron density. However, we find that the structure is more pronounced in the lighter positive ions than the heavy negative ions.

Figure 9 shows a power spectrum of the azimuthal electric field $E_{\theta}$, taken at a single grid point on the electron depletion boundary $\left(r / \lambda_{D e}=32\right)$. These waves are driven by plasma instabilities whose possible free energy sources we will discuss shortly. The spectrum is taken over the time period from $\omega_{\mathrm{pe}} t=200$ to 1000 and represents the waves in a nonlinear saturated state. The wave power is low frequency with the power existing between 0.1 to $0.3 \omega_{\text {LH }}$. Spectral analysis of the radial field, $E_{r}$, shows that it is essentially a zero-frequency (dc) field.

Wave growth similar to the case studied here was observed in numerical simulations by Sydora et al. [1983] and Galvez et al. [1988] of barium cloud releases in which the barium clouds were allowed to expand across a static magnetic field. The primary difference in these two studies was the fact that the work of Sydora et al. considered the case of a nonzero radial expansion velocity of the ions and electrons. Sydora et al. attributed these waves to the Kelvin-Helmholtz instability that results from the shear in the azimuthal flow velocity at the cloud boundary layer. The lower hybrid drift (LHD) instability resulting from $\mathbf{E} \times \mathbf{B}$ and $\nabla N \times \mathbf{B}$ drifts was

\section{POTENTIAL}

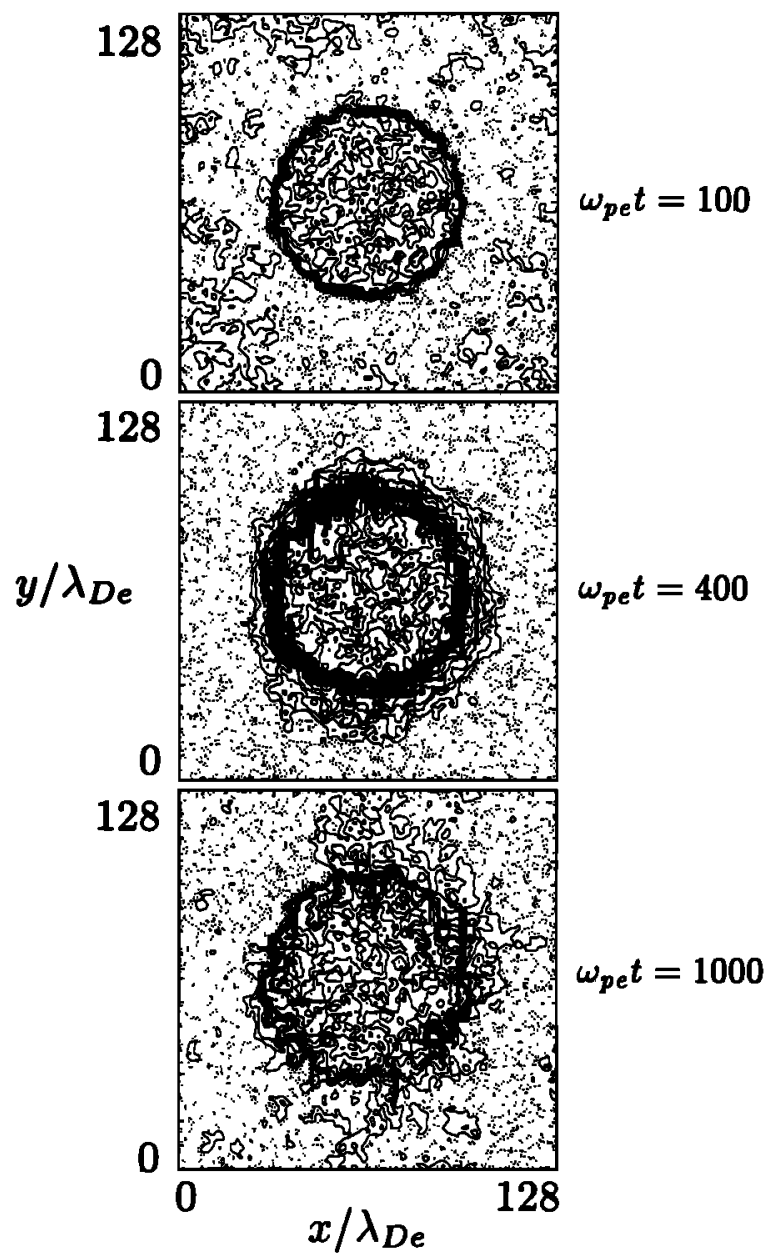

Figure 7. Potential $\left(e \phi / T_{e}\right)$ during the simulation run (contour levels run from -1.0 to 2.6 by 0.144 ). Dotted contours indicate $\phi<0$. Note the structure in the potential due to wave development.

argued to be the source of the waves observed in the simulation work of Galvez et al. Galvez et al. found that there was relatively good agreement between the wavelength of maximum growth obtained by solving the electrostatic dispersion relation for the LHD instability and the simulation results. The scaling of the instability wavelength with magnetic field strength $\left(\omega_{\mathrm{pe}} / \Omega_{\mathrm{ce}}\right)$ predicted by the LHD instability showed similar trends with the simulations. However, they found that the frequency and wavelength predicted by the LHD instability did not agree with the observations made during the AMPTE barium release experiments. In general, the predicted wavelengths were too short, and the predicted frequencies were too high. There was also disagreement between the frequency of the unstable waves and phase velocities predicted by the LHD instability and the results observed in the simulations. Galvez et al. alluded to the fact that these discrepancies could be due to their model's exclusion of the effect of shears in the electron flow velocity.

Another instability process that shows much promise in explaining the waves in the electron depletion bound- 


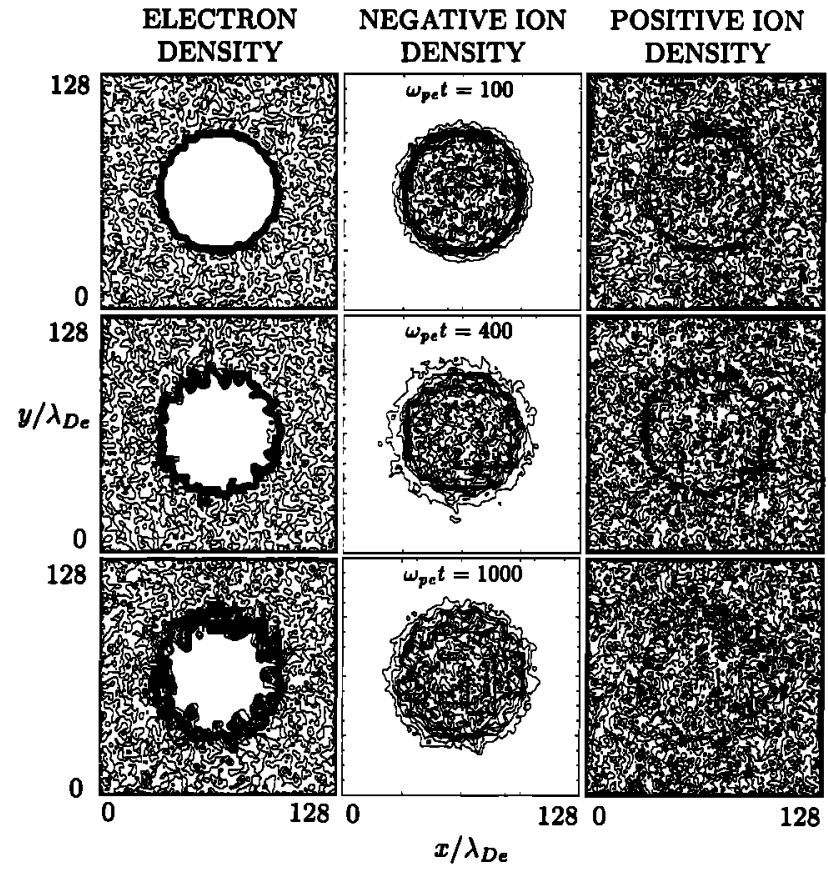

Figure 8. Densities of the three particle species during the run (contour levels run from 0.0 to 24.0 by 2.0 ). Note that pronounced structure develops in the electron density.

ary layer in our numerical simulations is the electronion-hybrid (EIH) instability of Ganguli et al. [1988]. This instability results from the shear in the electron flow velocity $V_{E}$. A nonlocal linear electrostatic fluid dispersion relation for waves propagating perpendicular to the magnetic field in a plasma with a perpendicular electron flow velocity and perpendicular flow velocity and density gradients is given by [Ganguli et al. 1988, 1993; Romero et al., 1992a]

$$
\left\{\frac{d^{2}}{d x^{2}}-k_{y}^{2}+G(\omega) \frac{k_{y}\left(V_{E}^{\prime \prime}(x)-\epsilon_{n} \Omega_{c e}\right)}{\omega-k_{y} V_{E}(x)}\right\} \phi_{1}(x)=0
$$

where $G(\omega)=\delta^{2} /\left(\delta^{2}+1\right)\left(1-\left(\omega_{\mathrm{LH}} / \omega\right)^{2}\right), \delta=\omega_{\mathrm{pe}} / \Omega_{\mathrm{ce}}$, $V_{E}^{\prime \prime}=d^{2} V_{E} / d x^{2}$ and $\epsilon_{n}=\left(d N_{e} / d x\right) / N_{e}$. The lower hybrid frequency $\omega_{\mathrm{LH}}$ is the value in the boundary layer where both ion species are present as discussed earlier, and the first-order perturbation electrostatic potential is denoted by $\phi_{1}(x)$. Note that in our simulation geometry, the radial variable $r$ corresponds to $x$ in (4), which is the direction of density and flow velocity gradients. The azimuthal variable $\theta$ corresponds to $y$, which is the direction of the flow velocity and wave propagation. The third term in (4) represents the free energy for wave growth. The two free energy sources are sheared electron flow $\left(V_{E}^{\prime \prime}(x)\right.$ and $\left.V_{E}(x)\right)$ and electron density gradients $\left(\epsilon_{n}\right)$. If the velocity shear contributions, $V_{E}^{\prime \prime}$ and $V_{E}$, are zero, then the dispersion relation in (4) reduces to the form for the LHD instability [Krall and Liewer 1971]. On the other hand, if the electron density gradient contribution $\epsilon_{n}$ is zero, then the dispersion relation reduces to that for the EIH instability [Ganguli et al., 1988; Romero et al., 1992a,b]. Both the LHD and EIH

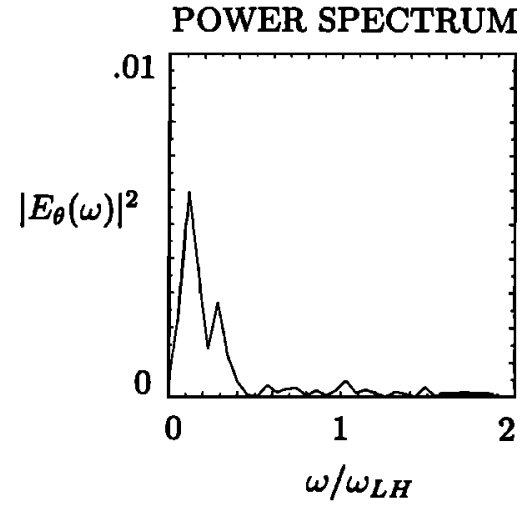

Figure 9. Power spectrum of the azimuthal electric field $E_{\theta}$, taken on the electron depletion boundary for $200<\omega_{\text {pe }} t<1000$.

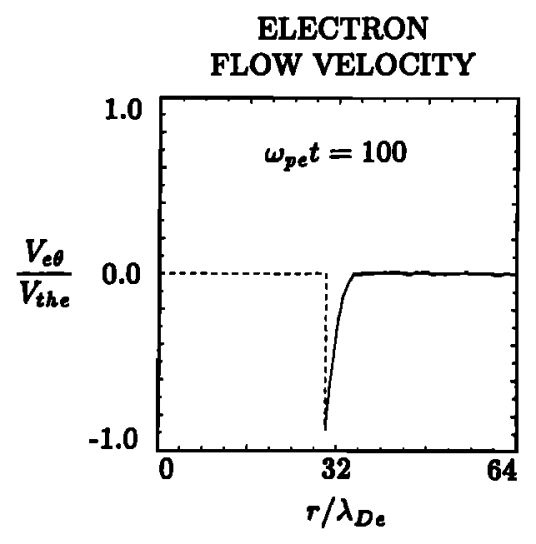

Figure 10. Azimuthal electron flow velocity profile at $\omega_{\text {pe }} t=100$, showing large initial velocity shears at the depletion boundary.

instabilities predict linear wave growth with $\omega \sim \omega_{L H}$. However, the EIH instability has the advantage in that it predicts longer wavelengths with $k_{\perp} L_{E} \sim 1$ where $k_{\perp}$ is the wavenumber perpendicular to the magnetic field and $L_{E}$ is the scale length of the electron flow velocity profile $\left(\rho_{e}<L_{E}<\rho_{0}\right)$. This is compared to $k_{\perp} \rho_{e} \sim\left(T_{e} / T_{i}\right)^{1 / 2}$ for the LHD instability. In general, both free energy sources in (4) may exist. However, the work of Romero et al. [1992b] has shown that if the shear frequency, $\omega_{B}=V_{\max } / L_{E}$, where $V_{\max }$ is the $\max$ imum value of the electron flow velocity, is larger than the lower hybrid frequency, $\omega_{L H}$, then the EIH instability driven by the electron flow velocity shears dominates the LHD instability.

To consider the role of the EIH instability in more detail, we show in Figure 10 the electron flow velocity profile early in the simulation $\left(\omega_{\mathrm{pe}} t=100\right)$ at the time waves initially begin to be observed in Figure 8. Figure 10 shows very large velocity shears near the depletion boundary. The maximum flow velocity is observed to be $-0.9 v_{\text {the }}$ and the scale length over which the electron flow is localized is roughly $2 \lambda_{\text {De }}$. This implies a shear frequency of $0.45 \omega_{\mathrm{pe}}$. The lower hybrid frequency from the parameters of section 2 is $0.134 \omega_{\text {pe }}$. Therefore $\omega_{\mathrm{s}} / \omega_{\mathrm{LH}}=3.4>1$, and the EIH instability should 
be the dominant source of the waves in our simulation run. The condition $k_{\perp} L_{E} \sim 1$ for EIH instabilitydriven waves predicts a wavelength of roughly $12.6 \lambda_{\mathrm{De}}$ which is in reasonable agreement with the value $9 \lambda_{\mathrm{De}}$ that is observed early during the simulation. Another important piece of evidence that supports the EIH instability is the nonlinear evolution of the system. As we discussed earlier, vortex formation is observed in the electron depletion boundary layer in the nonlinear stage of the development of the waves. This is the dominant nonlinear signature of the EIH instability $\left(\omega_{\mathrm{s}} / \omega_{\mathrm{LH}}>1\right)$, where the dominant nonlinear signature of the LHD instability $\left(\omega_{\mathrm{s}} / \omega_{\mathrm{LH}}<1\right)$ is the development of kinks in the electron density [Romero et al., 1992b]. Because of the broadening of the electron flow velocity profile by the EIH instability, the dynamical evolution of the system in time should exhibit a cascade from high frequencies $\left(\omega \sim \omega_{\mathrm{LH}}\right)$ and short wavelengths to lower frequencies $\left(\omega \ll \omega_{L H}\right)$ and longer wavelengths [Ganguli et al., 1993]. This is consistent with Figure 9 which shows that the power spectrum of the saturated state of the system is dominated by waves with frequencies that are much less than the lower hybrid frequency.

We have performed other simulation runs varying $T_{e} / T_{\mathbf{X}}, \omega_{\mathrm{pe}} / \Omega_{\mathrm{ce}}$, and $m_{e} / m_{\mathrm{o}}$. These show qualitatively similar results. We note that in varying $0.5<$ $\omega_{\mathrm{pe}} / \Omega_{\mathrm{ce}}<2$, we find that for stronger magnetic fields, the electron flow velocity profile scale length $L_{E}$ is shorter in the simulation runs. (The thickness of the negative charge surplus layer becomes thinner as the magnetic field strength increases as well.) We also find that the wavelength of the waves in the boundary layer are shorter in an absolute sense as the magnetic field strength increases. This is keeping with the fact that $k_{\perp} L_{E} \sim 1$ for the EIH instability.

\section{Summary and Conclusions}

Numerical simulations have been used to study the early-time evolution of electron density depletions and negative ion clouds produced during electron attachment chemical releases in the ionosphere. We have considered the dynamics in a plane perpendicular to the magnetic field and focused on timescales less than the negative ion cyclotron period. This is in contrast to previous simulation work that considered macroscopic fluid processes [Bernhardt, 1988; Scales and Bernhardt, 1991]. We find that a negative charge surplus develops outside the depletion initially as the negative ions move across the boundary, since they are unmagnetized on this timescale. The electrons remain bound to the field lines and are restricted from moving back into the depletion initially. This charge separation at the boundary produces a inhomogenous electric field across the boundary layer. A highly sheared electron flow velocity develops at the depletion boundary due to the $\mathbf{E} \times \mathbf{B}$ and $\nabla N \times \mathbf{B}$ drifts that result from this inhomogenous electric field and electron density gradients. We find that electrostatic waves begin to develop in the boundary after several lower hybrid periods and produce structuring in the boundary layer. Because of anomalous diffusion caused by these waves, the electrons may diffuse back into the depletion. This ultimately causes the density gradient and flow velocity profile to relax as well as a reduction in the electric field. We find that the wavelengths of these waves are less than the negative ion Larmor radius and that the frequency in the nonlinear regime to be much less than the lower hybrid frequency. The most likely candidate for this wave development is the EIH instability that results from shears in the electron flow velocity [Ganguli et al., 1988]. Shears in the electron flow velocity in the simulation runs were shown to be sufficiently large to excite the EIH instability. Also, the fact that vortices were observed in the nonlinear regime support the EIH mechanism.

Finally, we may briefly compare our results with waves observed during the NICARE 1 experiment. Our simulations describe the evolution on time periods of the order of or less than the negative ion cyclotron period. This corresponds to timescales of 0.2 seconds or less assuming $\mathrm{Br}^{-}$and $\mathrm{Ni}(\mathrm{CO})_{3}^{-}$cyclotron frequencies of 9 and $5 \mathrm{~Hz}$, respectively. Waves near $200 \mathrm{~Hz}$ were observed on this timescale as the diagnostic payload initially passed through the steep density gradients at the negative ion cloud boundary [Ganguli et al., 1993]. Assuming an ambient lower hybrid frequency in the kilohertz range, our power spectrum results would scale to frequencies in the hundreds of hertz which is in agreement with the observations. More detailed analysis of the electron density and waves observed during the NICARE series of experiments is now under investigation. Future work will make more detailed comparisons with the experimental data.

Our ongoing work is to incorporate and study the effects of electron attachment chemistry in our model. This study will provide a more realistic description of the attachment of the electrons and creation of negative ions by the released neutrals. A more accurate description of the initial formation of the electron and negative ion density gradients as well as the flow velocities in the boundary layer can be obtained from this model as well. Extending our simulation model to study lower-frequency waves at the negative ion cyclotron frequency is also under investigation to consider the waves at these frequencies observed at later times during the experiments.

Acknowledgments. This work was supported at Va. Tech by ONR grants N00014-92-J-1498 and N00014-92-J1484. The work was supported at NRL by the Office of Naval Technology Postdoctoral Fellowship Program (WAS) and NASA.

The Editor thanks a referee for his assistance in evaluating this paper.

\section{References}

Bernhardt, P. A., Chemistry and dynamics of $S F_{6}$ injections into the $F$ region, $J$. Geophys. Res., 89, 3929, 1984.

Bernhardt, P. A., E. J. Weber, J. G. Moore, J. Baumgardner, and M. Mendillo, Excitation of oxygen permitted line 
emissions by $S F_{B}$ injection into the $F$ region, $J$. Geophys. Res., 91, 8937, 1986.

Bernhardt, P. A., and L. M. Duncan, Ionospheric focused heating - A hybrid modification experiment, J. Atmos. Terr. Phys., 49, 1107, 1987 a.

Bernhardt, P. A., A critical comparison of ionospheric depletion chemicals, J. Geophys. Res., 92, 4617, $1987 \mathrm{~b}$.

Bernhardt, P. A., Cross-B convection of artificially created, negative-ion clouds and plasma depressions: Low-speed flow, J. Geophys. Res., 93, 8696, 1988.

Bernhardt, P. A., P. Rodriquez, C. L. Siefering, and C. S. Lin, Field-aligned dynamics of chemically induced perturbations to the ionosphere, J. Geophys. Res., 96, 13,887, 1991.

Bernhardt, P. A., J. D. Huba, J. A. Fulford, P. A. Forsyth, D. N. Anderson, and S. T. Zalesak, Analysis of rocket beacon transmissions to reconstruct ionospheric densities, Radio Sci., 28, 613, 1993.

Birdsall, C. K., and A. B. Langdon, Plasma Physics Via Computer Simulation, Adam Hilger, New York, 1991.

Galvez, M., S. P. Gary, C. Barnes, and D. Winske, Computer simulations of plasma expansion across a magnetic field, Phys. Fluids, 31, 1554, 1988.

Ganguli, G., Y. C. Lee, and P. Palmadesso, Electron-ion hybrid mode due to transverse velocity shear, Phys. Fluids, 31, 2753, 1988.

Ganguli, G., P. A. Bernhardt, W. Scales, P. Rodriquez, C. L. Siefering, and H. Romero, Physics of negative ion plasmas created by chemical releases in space, in Physics of Space Plasmas (1992), Scientific Publishers. Conf. Proc. Repr. Ser., vol. 12, Scientific Publishers, Cambridge, MA, p. $161,1993$.
Hockney, R. W., and J. W. Eastwood, Computer Simulation Using Particles, Adam Hilger, New York, 1988.

Krall, N. A., and P. C. Liewer, Low-frequency instabilities in magnetic pulses, Phys. Rev. A: Gen. Phys., 4, 2094, 1971.

Mendillo, M., and D. Forbes, Theory and observations of a dynamically evolving negative ion plasma, J. Geophys. Res., 87, 8273, 1982.

Romero, H., G. Ganguli, Y. C. Lee, and P. J. Palmadesso, Electron-ion hybrid instabilities driven by velocity shear in a magnetized plasma, Phys. Fluids $B, 4,1708,1992 a$.

Romero, H., G. Ganguli, and Y. C. Lee, Ion acceleration and coherent structures generated by lower hybrid sheardriven instabilities, Phys. Rev. Lett., 69, 3503, 1992b.

Scales, W. A., and P. A. Bernhardt, Simulation of highspeed (orbital) releases of electron attachment materials in the ionosphere, J. Geophys. Res., 96, 13,815, 1991.

Sydora, R. D., J. S. Wagner, L. C. Lee, and E. M. Wescott, Electrostatic Kelvin-Helmholtz instability in a radially injected plasma cloud, Phys. Fluids, 26, 2986, 1983.

Tajima, T., Computational Plasma Physics: With Applications to Fusion and Astrophysics, Addison Wesley, New York, 1989.

P. A. Bernhardt and G. Ganguli, Space Plasma Branch, Plasma Physics Division, Naval Research Laboratory, Washington, D. C. 20375. (e-mail: bern@ppd.nrl.navy.mil)

W. A. Scales, Bradley Department of Electrical Engineering, Virginia Tech, Blacksburg, VA 24061. (e-mail: wayne@starchild.ee.vt.edu)

(Received April 22, 1993; revised August 18, 1993; accepted September 17, 1993.) 